\title{
A SPECIAL CONGRUENCE
}

L. CARLITZ

1. It is familiar that if $p$ is a prime such that $p-1\left\{m, p^{r} \mid m\right.$ then

$$
B_{m} \equiv 0\left(\bmod p^{r}\right) \text {, }
$$

where $B_{m}$ denotes a Bernoulli number in the even suffix notation. The writer has recently proved the companion formula ([2, Theorem 3$]$; see also [1])

$$
B_{m(p-1)}+1 / p-1 \equiv 0\left(\bmod p^{r}\right) \quad(p \geqq 3),
$$

for $p^{r} \mid m, m>0$; moreover if $m=p^{r} h$, then

$$
p^{-r}\left(B_{m(p-1)}+1 / p-1\right) \equiv h w_{p}(\bmod p) \quad(p>3),
$$

where $w_{p}$ denotes Wilson's quotient $((p-1) !+1) / p$.

In this note we show that the above formulas imply

$$
p+(p-1) \sum_{0<s(p-1)<m}\left(\begin{array}{c}
m \\
s(p-1)
\end{array}\right) \equiv 0\left(\bmod p^{r+1}\right),
$$

where $p^{r} \mid m$ and $p \geqq 3$. More precisely if $m=p^{r} m_{0}$, we have, for $p>3$,

$$
\begin{aligned}
& p^{-r-1}\left\{p+(p-1) \sum_{0<s(p-1)<m}\left(\begin{array}{c}
m \\
s(p-1)
\end{array}\right)\right\} \\
& \equiv m_{0}\left\{\frac{1}{2}-\sum_{0<2 s<m, p-1 / 2 s}\left(\begin{array}{c}
m-1 \\
2 s-1
\end{array}\right) \frac{B_{2 s}}{2 s}+\delta_{m} \frac{w_{p}}{p-1}\right\} \quad(\bmod p),
\end{aligned}
$$

where $\delta_{m}=1$ for $p-1 \mid m-1, \delta_{m}=0$ otherwise.

For $r=0,(1.4)$ is due to Hermite. The proof below of (1.4) was suggested by Nielsen's proof [3, p. 254] of Hermite's formula.

2. Proof of (1.4). Using the basic recurrence for the Bernoulli numbers we may write

$$
1-\frac{1}{2} m+\sum_{0<2 s<m}\left(\begin{array}{c}
m \\
2 s
\end{array}\right) B_{2 s}=0 .
$$

Now let $p^{r} \mid m$. Consider first a term such that $p-1 \nmid 2 s$. Let $p^{k} \mid s$, so that by $(1.1), B_{2 s} \equiv 0\left(\bmod p^{k}\right)$. If $k \leqq r$, it follows that

$$
\left(\begin{array}{c}
m \\
2 s
\end{array}\right)=\frac{m}{2 s}\left(\begin{array}{c}
m-1 \\
2 s-1
\end{array}\right) \equiv 0\left(\bmod p^{r-k}\right)
$$

Received by the editors February 2, 1953. 
and consequently

$$
\left(\begin{array}{l}
m \\
2 s
\end{array}\right) B_{2 s}=0\left(\bmod p^{r}\right) \text {. }
$$

Clearly (2.3) holds also for $k>r$. Thus (2.1) and (2.2) imply

$$
1+\sum_{0<s(p-1)<m}\left(\begin{array}{c}
m \\
s(p-1)
\end{array}\right) B_{s(p-1)} \equiv 0\left(\bmod p^{r}\right),
$$

which may be rewritten as

$$
\begin{aligned}
1+\sum_{0<s(p-1)<m}\left(\begin{array}{c}
m \\
s(p-1)
\end{array}\right)\left(B_{s(p-1)}+\frac{1}{p}-1\right) \\
\quad \equiv\left(\frac{1}{p}-1\right) \sum_{0<s(p-1)<m}\left(\begin{array}{c}
m \\
s(p-1)
\end{array}\right)\left(\bmod p^{r}\right) .
\end{aligned}
$$

Now exactly as in proving (2.3), we may show, using (1.2), that

$$
\left(\begin{array}{c}
m \\
s(p-1)
\end{array}\right)\left(B_{a(p-1)}+\frac{1}{p}-1\right) \equiv 0\left(\bmod p^{r}\right) .
$$

Thus (2.4) reduces to

$$
1 \equiv\left(\frac{1}{p}-1\right) \sum_{0<8(p-1)<m}\left(\begin{array}{c}
m \\
s(p-1)
\end{array}\right)\left(\bmod p^{r}\right) .
$$

It is evident that (2.5) and (1.4) are equivalent.

3. Proof of (1.5). We again begin with (2.1) which we now write as

$$
\begin{aligned}
1-\frac{1}{2} m+\sum_{0<2 s<m, p-1 / 2 s}\left(\begin{array}{c}
m \\
2 s
\end{array}\right) B_{2 s} & \\
& +\sum_{0<s(p-1)<m}\left(\begin{array}{c}
m \\
s(p-1)
\end{array}\right) B_{s(p-1)}=0 .
\end{aligned}
$$

This evidently implies

$$
\begin{aligned}
1-\frac{1}{2} m+ & \sum_{0<2 s<m, p-1 \mid 2 s}\left(\begin{array}{c}
m \\
2 s
\end{array}\right) B_{2 s} \\
& \quad+\sum_{0<s(p-1)<m}\left(\begin{array}{c}
m \\
s(p-1)
\end{array}\right)\left(B_{s(p-1)}+\frac{1}{p}-1\right) \\
& =\sum_{0<s(p-1)<m}\left(\begin{array}{c}
m \\
s(p-1)
\end{array}\right)\left(\frac{1}{p}-1\right) .
\end{aligned}
$$


Consider first the sum

$$
S=\sum_{0<s(p-1)<m}\left(\begin{array}{c}
m \\
s(p-1)
\end{array}\right)\left(B_{s(p-1)}+\frac{1}{p}-1\right) .
$$

Let $p^{k} \mid s$ and put

$$
s=p^{k} h
$$

then by (1.3) we have

$$
B_{s(p-1)}+\frac{1}{p}-1 \equiv p^{k} h w_{p}\left(\bmod p^{k+1}\right) .
$$

If $k \leqq r$ it is evident from (2.2) that (3.3) yields

$$
\begin{aligned}
\left(\begin{array}{c}
m \\
s(p-1)
\end{array}\right)\left(B_{s(p-1)}+\frac{1}{p}-1\right) & \\
& \equiv\left(\begin{array}{c}
m \\
s(p-1)
\end{array}\right) p^{k} h w_{p}\left(\bmod p^{r+1}\right) ;
\end{aligned}
$$

clearly (3.4) holds also for $k>r$. Since the right member of (3.4) is equal to

$$
m\left(\begin{array}{c}
m-1 \\
s(p-1)-1
\end{array}\right) w_{p} /(p-1)
$$

we see that (3.2) becomes

$$
S \equiv \frac{m w_{p}}{p-1} \sum_{0<s(p-1)<m}\left(\begin{array}{c}
m-1 \\
s(p-1)-1
\end{array}\right)\left(\bmod p^{r+1}\right) .
$$

In the next place for the first sum in the left member of (3.1) we have

$$
\sum_{0<2 s<m, p-1 / 2 s}\left(\begin{array}{c}
m \\
2 s
\end{array}\right) B_{2 s}=m \sum_{0<2 s<m, p-1 / 2 s}\left(\begin{array}{c}
m-1 \\
2 s-1
\end{array}\right) \frac{B_{2 s}}{2 s} .
$$

Substituting from (3.5) and (3.6) in (3.1) we get

$$
\begin{aligned}
1-\left(\frac{1}{p}-1\right) & \sum_{0<s(p-1)<m}\left(\begin{array}{c}
m \\
s(p-1)
\end{array}\right) \\
\equiv & \frac{1}{2} m-m \sum_{0<2 s<m, p-1 / 2 s}\left(\begin{array}{c}
m-1 \\
2 s-1
\end{array}\right) \frac{B_{2 s}}{2 s} \\
& \quad-\frac{m w_{p}}{p-1} \sum_{0<s(p-1)<m}\left(\begin{array}{c}
m-1 \\
s(p-1)-1
\end{array}\right)\left(\bmod p^{r+1}\right) .
\end{aligned}
$$


Now let $p^{r}\left|m, p^{r+1}\right| m$; then (3.7) becomes

$$
\begin{aligned}
\frac{1}{m}\left\{1-\left(\frac{1}{p}-1\right)\right. & \left.\sum_{0<s(p-1)<m}\left(\begin{array}{c}
m \\
s(p-1)
\end{array}\right)\right\} \\
\equiv & \frac{1}{2}-\sum_{0<2 s<m, p-1 / 2 s}\left(\begin{array}{c}
m-1 \\
2 s-1
\end{array}\right) \frac{B_{2 s}}{2 s} \\
& -\frac{w_{p}}{p-1} \sum_{0<s(p-1)<m}\left(\begin{array}{c}
m-1 \\
s(p-1)-1
\end{array}\right)(\bmod p) .
\end{aligned}
$$

But $[3$, p. 255]

$$
\sum_{0<s(p-1)<m}\left(\begin{array}{c}
m-1 \\
s(p-1)-1
\end{array}\right) \equiv\left\{\begin{aligned}
0 & (p-1 \nmid m-1) \\
-1 & (p-1 \mid m-1)
\end{aligned}\right.
$$

indeed (3.9) is an easy consequence of the case $r=0$ of (1.4). Finally (3.7), (3.8), and (3.9) evidently imply (1.5).

\section{REFERENCES}

1. L. Carlitz, $A$ divisibility property of the Bernoulli number, Proc. Amer. Math. Soc. vol. 3 (1952) pp. 604-607.

2. - Some congruences for the Bernoulli numbers, Amer. J. Math. vol. 75 (1953) pp. 163-172.

3. N. Nielsen, Sur le theorime de v. Staudt et de Th. Clausen relatif aux nombres de Bernoulli, Annali di Matematica (3) vol. 22 (1914).

DUKE UNIVERSITY 